\title{
Zum Schwarmverhalten der Sizilianischen Honigbiene Apis mellifera sicula (Montagano 1911)
}

\author{
K Tiemann, D Brückner * \\ Forschungsstelle für Bienenkunde, Universität Bremen, Postfach 330440, \\ W-2800 Bremen 33, Deutschland
}

(Eingegangen 25 Oktober 1992; angenommen 3 Februar 1993)

\begin{abstract}
Zusammenfassung - Das Schwarmverhalten der Rasse Apis mellifera sicula wurde in 9 Völkern in Westsizilien untersucht. Die Kolonien produzieren eine große Anzahl an Weiselzellen und es schlüpfen bis zu 33 Königinnen täglich. Die Schwärme sind immer polygyn. Die virginellen Königinnen koexistieren im Volk und in der neuen. Kolonie meist bis zu einem Lebensalter von 2 bis 3 Tagen. Die Änderung im Aggressionsverhalten beider Kasten mit zunehmendem Alter der Königinnen scheint von Pheromonen bestimmt zu sein und tritt vor dem Paarungsflug auf. Das Ausgangsvolk produziert solange Schwärme, bis nur ein kleines Restvolk zurückbleibt.
\end{abstract}

Apis mellifera sicula / Schwarmverhalten / Polygynie

\section{EINLEITUNG}

Das natürliche Vorkommen der Apis mellifera sicula beschränkt sich auf Sizilien. Eine komplette Isolation liegt durch die Insellage nicht vor, die Distanz von Kalabrien, dem italienischen Festland, bis nach Sizilien kann von Bienen überflogen werden. Die sizilianische Honigbiene ist zierlich und dunkel. Sie kann auf ihren Tergiten zwar gelbe Flecken aufweisen, doch nie eine gelbe Bande des ersten Abdominaltergits (Ruttner, 1988; KauhausenKeller, 191).
In ihrer reinrassigen Form ist die sizilianische Biene sanftmütig und kann viel Honig eintragen. Nach eigenen Beobachtungen findet diese Biene in norddeutschen Gebieten noch Nektar, wenn der Imker bei der hiesigen Rasse (Apis mellifera carnica) schon zufüttern muß. Beim Arbeiten mit der $A m$ sicula zeigt sich ihr nervöses Temperament und eine höhere Angriffslust, die nicht unbedingt mit Stechlust gleichzusetzen ist. Die sizilianische Biene ist eng an das mediterrane Klima Siziliens mit den heißen trockenen Sommern adaptiert. Ein Brutnest und Drohnen werden das ganze Jahr über gehalten. Die

\footnotetext{
* Korrespondenz und Sonderdrucke
} 
Brutentwicklung wird nur im heißen Sommer im Juli/August reduziert. Beim Auftreten des heißen Saharawindes zu Beginn der Trockenzeit können die Bienen ihre Flugtätigkeit für einige Tage auch völlig einstellen.

Die sizilianische Honigbiene zeigt ein für europäische Rassen außergewöhnliches Reproduktionsverhalten. Sie produziert in der Schwarmzeit, die im Februar/ März mit der Mandelblüte beginnt, eine enorme Anzahl an Weiselzellen. Bei starken Völkern sind es über 200 verdeckelte Königinnenzellen geichzeitig.

Im Vergleich dazu produziert die, allerdings auf Schwarmträgheit gezüchtete, A $m$ carnica 6 bis 7 verdeckelte Weiselzellen an einem Tag. Die in Deutschland verbreitete $A m$ carnica schwärmt sobald die erste Weiselzelle verdeckelt ist und der erste Nachschwarm folgt nach dem Schlupf der ersten Jungkönigin, meist 7 Tage später (Zander und Weiß, 1964). Eine Koexistenz von Königinnen ist bei dieser Bienenrasse nur in Ausnahmefällen bekannt. Bei den sizilianischen Bienenvölkern wurde beobachtet, daß vor dem Schwärmen, im Schwarm und auch in der neuen Kolonie mehrere Königinnen koexistieren, möglicherweise bis nach dem Paarungsflug (Ruttner, 1988). Die sizilianische Honigbiene zeigt demnach ein polygynes Verhalten, was von den 24 Rassen der Art Apis mellifera nur noch drei weitere mediterrane Rassen zeigen ( $A m$ syriaca, $A$ m lamarckii und $A$ m intermissa). Für die Rasse $A m$ lamarckii beschreibt ButtelReepen (1921) Polygynie in den Vor- und Nachschwärmen.

Die vorliegende Arbeit wurde durchgeführt, um das Polygynieverhalten $\operatorname{der} A \mathrm{~m}$ sicula im Freiland detailliert zu untersuchen. Dabei sollten folgende Fragen geklärt werden:

- Wieviele Königinnen koexistieren im alten Volk, im Schwarm und im neuen Volk?
- Wie ist die Altersstruktur der koexistierenden Königinnen?

- Wie ist das Aggressionsverhalten der Stockmutter, der virginellen Königinnen und der Arbeiterinnen zueinander?

- Wie lange koexistieren die virginellen Königinnen in der neuen Kolonie?

\section{MATERIAL UND METHODEN}

Die Versuche wurden wegen des kalten Frühjahres 1992 erst relativ spät vom 16.3 bis 11.4.1992, in Trapani (Westsizilien) durchgeführt.

Für die Versuche standen 9 Bienenvölker zur Verfügung. Die Völker wurden aufgrund ihrer dunklen Farbmorphe und Gestalt ausgewählt. Bei der ersten Durchsicht am 16.3.1992 wurde deren Entwickłung zur Schwarmtätigkeit autgrund der Anzahl der angesetzten Weiselzellen festgestellt. Die eierlegende Königin wurde gesucht und mit einer Nummer versehen. Alle 9 Völker waren mehr oder weniger gleich stark, standen auf einer Zarge (Rähmchenmaß Dadant, Wabenzahl 10) und waren dicht besetzt. Die Bienenvölker wurden, zusätzlich zum natürlichen Nahrungsangebot, gefüttert. Durch dieses Überangebot an Futter und die Raumnot sollte die Schwarmtätigkeit zusätzlich angeregt werden (Winston 1987, 190-197). Alle Völker wurden auf einem sonnigen, windgeschützten Platz aufgestellt. Die Schwarmentwicklung war trotz gleicher Volksstärke sehr unterschiedlich. Einige Völker hatten schon lang ausgezogene Weiselzellen, die bald verdeckelt wurden, andere hatten noch keine Weiselzellen angesetzt.

Bei der Kontrolle der Völker wurden die verdeckelten Weiselzellen auf jeder Wabe gezählt. In jedem Volk wurde nach geschlüptten Königinnen gesucht, jede neu geschlüptte Königin wurde entnommen, mit numerierten Opalithplättchen individuell markiert und auf der gleichen Wabe dem Volk wieder zugesetzt. Dadurch konnte eine Aussage über inre Überlebensdauer im Volk gemacht werden. Zusätzlich wurde vermekt, ob sich die Königinnen dicht nebeneinander auf einer Wabe aufhielten oder ein bestimmtes Areal für sich beanspruchten. Die Stockmutter wurde täglich gesucht und ihre Ei- 
produktion und Schwarmbereitschaft protokolliert. Aus arbeitstechnischen Gründen konnte nicht jedes Volk täglich auf alle Parameter hin kontrolliert werden.

Vor jeden Bienenkasten, ob es das Ausgangsvolk oder eine geschwärmte neue Kolonie war, wurde eine Folie in einer Breite von $1 \mathrm{~m} \mathrm{ge-}$ spannt. Um diese Fläche wurde eine $10 \mathrm{~cm}$ hohe Umrandung aus gewelltem Plastik gesetzt. Über diese Konstruktion wurde dann noch ein Netz gespannt. Mit dieser Netzfläche war es möglich, die abgestochenen Königinnen, die von den Arbeiterinnen aus dem Stock heraustransportiert werden, zusätzlich zu erfassen. Die Umrandung der Fläche zwang die Bienen, mit ihrer schweren Last hochzufliegen, was erfahrungsgemäß ein Fallenlassen der toten Königinnen bewirkt. So war eine vollständige Erfassung dieser vor dem Bienenstock möglich. Das Netz über der Folienfläche verhinderte ein Absammein der toten Bienen durch Vögel. Die Neztflächen wurden täglich kontrolliert.

Bei der Durchsicht der Völker wurde das Verhalten der Arbeiterinnen zu den geschlüptten Königinnen beurteilt. Waren sie uninteressiert an den Königinnen, interessiert oder aggressiv? Wenn eine Königin für die Bienen uninteressant ist, so kann sie ungehindert über die Waben laufen, ihre motorische Aktivität wirkt nicht gehetzt oder aufgeregt. Sind die Bienen an der Königin interessiert, so wird sie mit den Antennen betastet oder geleckt, und eine häufige Futterübergabe ist zu beobachten. Bei Aggressionen zwischen Königin und Arbeiterinnen wird diese verfolgt und mit den Mandibeln festgehalten. Die Bienen sind oft in Stechhaltung und sie bilden häufig ein dichtes Knäuel um die Königin.

Die aus den Völkern abgehenden Schwärme wurden gezählt und die während des Schwarmprozesses neu geschlüpfen Königinnen in der Schwarmtraube oder nach dem Einfangen des Schwarmes gekennzeichnet. Das Durchsieben der gesamten Schwärme hatte sich, bedingt durch die damit entstehende Unruhe, für die A $m$ sicula nicht bewährt. Das Verhalten der Königinnen und der Arbeiterinnen im Schwarm wurde beobachet. Hatte der Schwarm sich beruhigt und bildete eine feste Schwarmtraube, so wurde er in einen Kasten eingefangen. Die eingefangenen Schwärme wurden einen Tag ungestört gelassen und am zweiten Tag nach den Königinnen durchsucht. Ihr Alter wurde notiert sowie ihr Verhalten und das der Arbeiterinnen beobachtet.

\section{ERGEBNISSE}

\section{Weiselzellenproduktion im Bienenvolk}

Die Weiselzellenproduktion konnte in 2 Völkern kontinuierlich verfolgt werden, da nur in diesen die Königinnen in Eilage blieben (s unter 4). Weitere 2 Völker starteten trotz hoher Volksstärke und zusätzlicher Futtergabe nicht mit der Weiselzellproduktion.

Beide auf ihre Weiselzellproduktion untersuchten Völker starteten mit ungefähr der gleichen Anzahl an verdeckelten Weiselzellen, allerdings mit 4 Tagen Abstand. Die Weiselzelienproduktion stieg dann bis zu einem Maximum kontinuierlich an. Die höchste Anzahl an verdeckelten Weiselzellen betrug 232 Zellen an einem Tag (Abb 1, Volk 40 am 8. April).

\section{Anzahl der Königinnen im alten Volk}

Bedingt durch die schlechte Wetterlage, konnte in 6 Völkern die Überlebensdauer der Königinnen im Stock eine Woche lang beobachtet werden. Bei wärmerer, windstiller Witterung wäre der größte Teil dieser Königinnen mit den Arbeiterinnen ausgeschwärmt. Im Gegensatz zu Völkern anderer europäischen Rassen schlüpften in den beiden bis zum Abgang des Vorschwarms beobachteten Völkern die Junköniginnen schon vor dem Schwarm. Siebzehn Königinnen lebten nicht länger als einen Tag zusammen im Volk, weitere 17 lebten nicht länger als 2 Tage im Volk (Abb 2). Nur eine geschlüpfte Königin hatte eine Überlebensdauer von 5 Tagen. Von 35 markierten Königinnen waren demnach 34 nach 2 Tagen abgestochen.

Die Anzahl der geschlüpften Königinnen pro Tag variierte in den 2 untersuchten Völkern beträchtlich (von 1 bis 33). Jeweils 


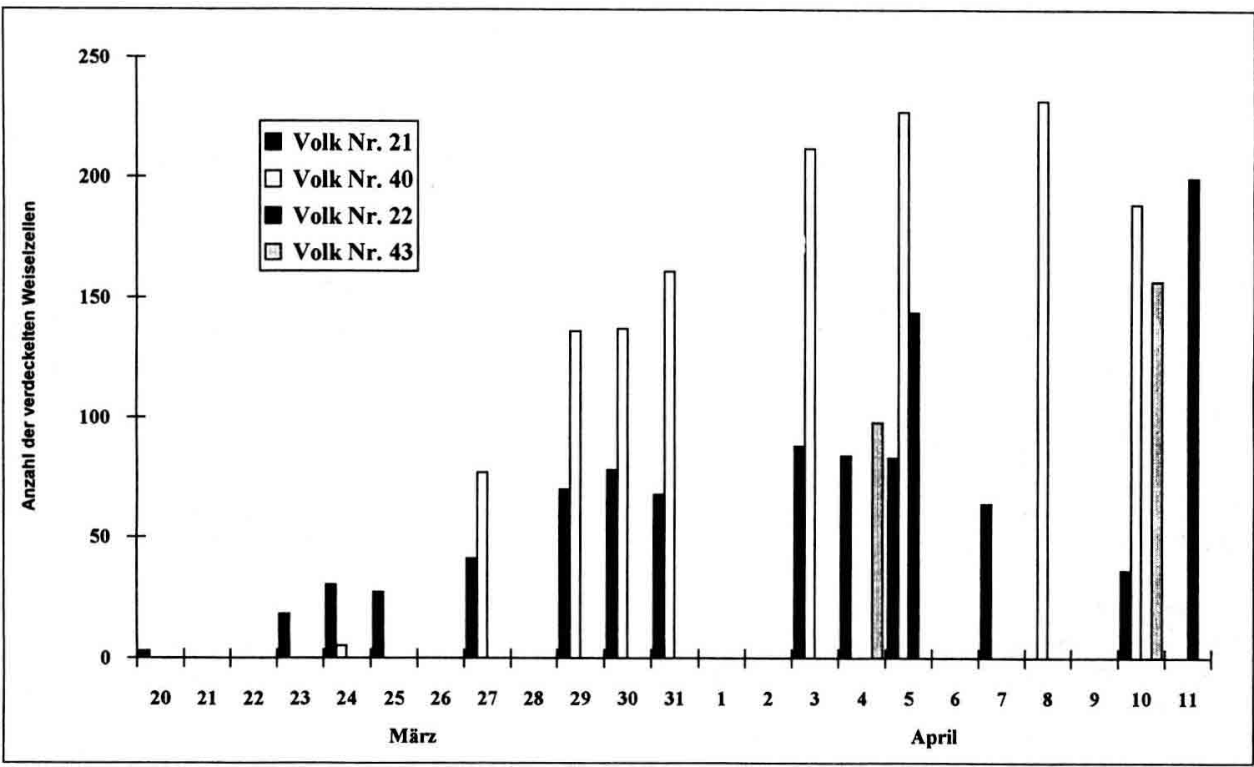

Abb 1. Anzahl (absolut) der verdeckelten Weiselzellen in den weiselrichtigen Völkern Die Anzahl der verdeckelten Weiselzellen ist für die 4 Völker als Säulen dargestellt. Die Kontrolle der Weiselzellen konnte aus arbeitstechnischen Gründen nicht für jedes Volk an jedem der oben angegebenen Beobachtungstagen erfolgen.

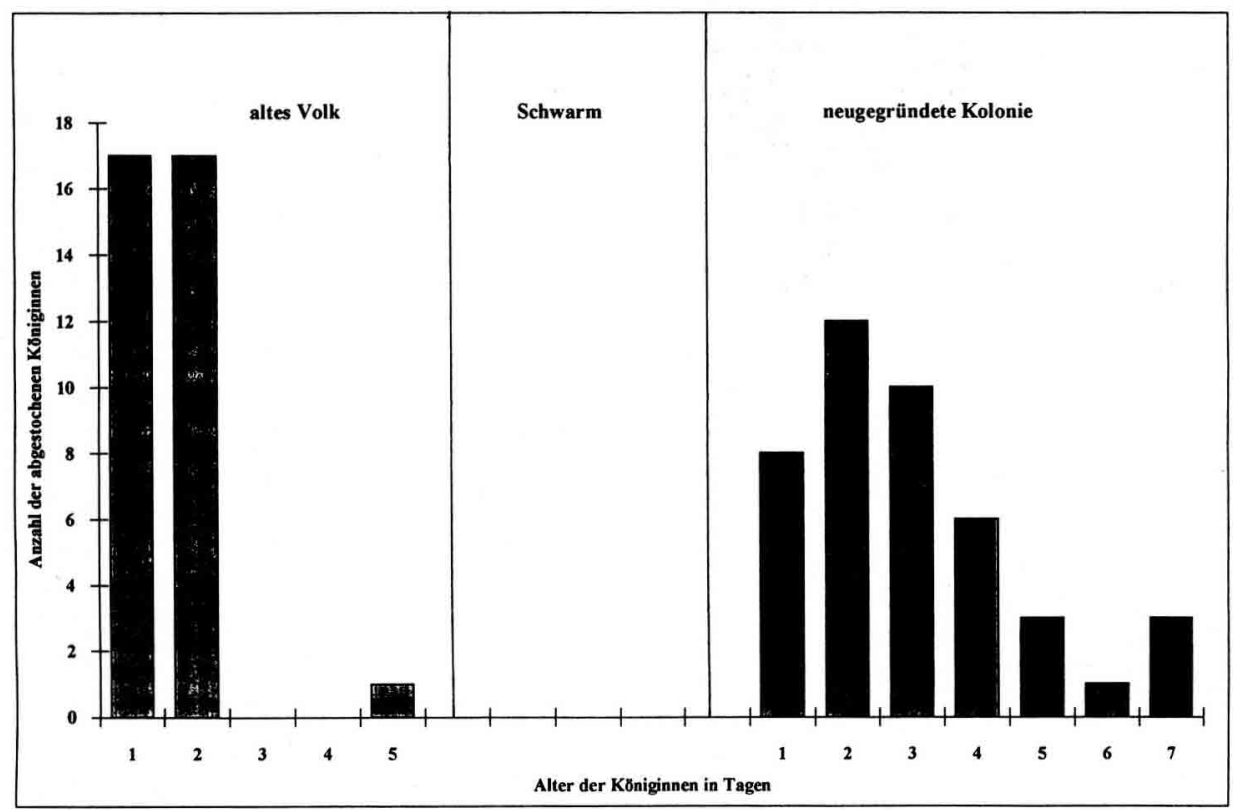

Abb 2. Überlebensdauer der Königinnen im alten Volk, im Schwarm und in der neuen Kolonie. 
am Schwarmtag wurden die höchsten Individuenzahlen beobachtet: Als Beispiel schlüpften am $2.4 \mathrm{im}$ Volk 21 zugleich 10 Königinnen, am 5.4 vor dem nächsten Schwarm in diesem Volk schlüpften 12 Königinnen. Zwischen den Schwärmen ging die Anzahl der geschlüpften Königinnen auf 5 oder weniger Individuen zurück. Es scheinen viele Königinnen während des Schwärmens verloren zu gehen, denn die Anzahl der geschlüpften Königinnen zu den tatsächlich im eingefangenen Schwarm gefundenen variiert stark.

Das Verhalten der jungen Königinnen untereinander ist sehr unterschiedlich. Nach dem Schlupf sind sie oft bis zu einem Tag auf einer Wabe zusammen zu finden. Ihre motorische Aktivität ist sehr hoch und beruhigt sich, wenn die Königinnen sich auf getrennten Waben befinden.

\section{Verhalten der Arbeiterinnen zu den virginellen Königinnen im Volk}

Die Affinität zu den virginellen Königinnen scheint im Volk nicht sehr hoch. Sie wurden gefüttert, aber nicht deutlich geleckt oder betastet. Bei schlechtem Wetter geschah eine 'Geburtenkontrolle' durch Ausbeißen der verdeckelten Weiselzellen. Starke Aggressionen zwischen Arbeiterinnen und Königinnen konnten nicht beobachtet werden, wohl aber Aggressionen zwischen den Königinnen. Selbst fremde virginelle Königinnen, die in den Stock über das Flugloch zuliefen, wurden von den Arbeiterinnen toleriert.

\section{Der Zustand der Stockmutter}

In 5 von 9 Bienenvölkern ging die Stockmutter schon vor dem Schwärmen verloren. In einem Volk wurde zweimalig ein
Schwarmversuch mit der alten Königin unternommen, wobei diese aber nicht fliegen konnte. Sie wurde daraufhin für die Experimente entfernt, um Nachschwärme zu ermöglichen. Letztendlich ist nur eine alte Königin geschwärmt, welche ihre Eilage erst einen Tag vor dem Abschwärmen einstellte. Zwei Tage nach dem Schwärmen war sie bereits wieder in Eilage. Wieweit die häufigen Inspektionen die Ursache für die hohen Königinnenverluste bilden, kann nicht entschieden werden.

\section{Schwarm und neue Kolonien}

Wir beobachteten 20 Schwärme, die unterschiedliches Schwarmverhalten zeigten: Schwärme aus unterschiedlichen Völkern setzten sich zusammen an einen Platz und bildeten eine große gemeinsame Schwarmtraube. Aus einem Volk konnten mehrere Schwärme hintereinander abgegeben werden und diese konnten sich an getrennten Stellen zur Schwarmtraube niederlassen. Es wurden Schwärme beobachtet, die in andere Bienenkästen hineinschwärmten, dort ein paar Tage toleriert wurden und dann wieder ausschwärmten. Zusätzlich flogen Schwärme zurück in den eigenen Bienenkasten und schwärmten nach einigen Stunden nochmals.

Alle von uns beobachteten Schwärme waren polygyn. Es wurden 2 (der einzige Vorschwarm) bis 13 Königinnen je Schwarm gezählt ( $\mathrm{Abb} 3$ ). In den neuen Kolonien veränderte sich die Situation (Abb 2). In diesem Histogramm zeigt sich ein deutliches Maximum in der Anzahl der abgestochenen Königinnen nach 2 Tagen. In 30 von 43 Fällen (fast $70 \%$ ) ist die Überlebensdauer im neuen Volk auf 2 bis 3 Tage begrenzt. Vor dem Paarungsflug, im Alter von 6-10 Tagen, sind fast alle neuen Kolonien wieder monogyn. 


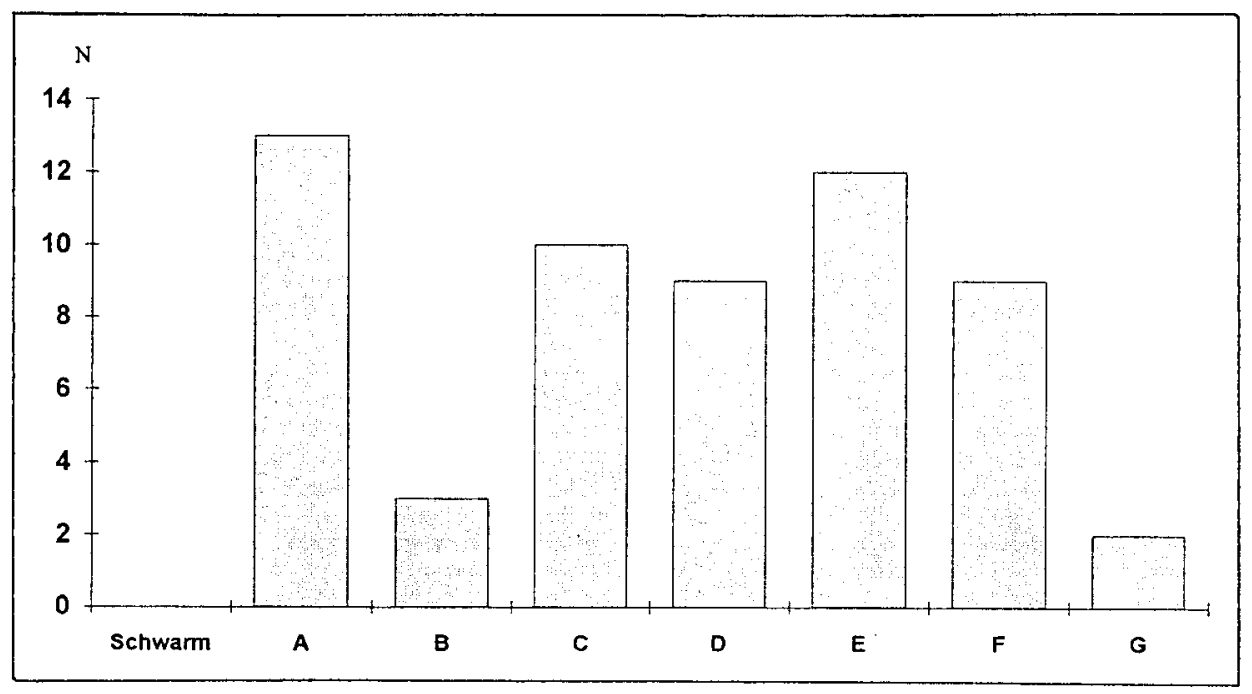

Abb 3. Anzahl der Königinnen in den eingeschlagenen Schwärmen.

\section{Verhalten der Arbeiterinnen zu den virginellen Königinnen im Schwarm und der neuen Kolonie}

Während des Schwärmens und in der Schwarmtraube zeigte sich eine geringe Affinität zwischen den Arbeiterinnen und den Königinnen. Königinnen, die aus der Schwarmtraube herausfielen, wurden nicht weiter beachtet. Wurden sie wieder zugesetzt oder flogen sie selber wieder dorthin, wurden sie weiterhin toleriert und nicht abgestochen. Die Bienen bilden erst bei mehr als 3 Königinnen eine feste Schwarmtraube; sind weniger Königinnen in der Traube, auch wenn eine alte Königin dabei ist, so fliegen die Arbeiterinnen wieder zurück zum alten Stock. So fliegt ein Schwarm oft mehrmals hin und her, weil immer wieder Königinnen auf dem Flug verloren gehen. Aggressionen zwischen den Königinnen wurden in der
Schwarmtraube nicht gefunden. Das Verhalten der Arbeiterinnen zu den Königinnen ändert sich deutlich beim eingefangenen Schwarm. Bei der ersten Durchsicht, nach 2 Tagen, sind Knäule von Bienen zu beobachten, in deren Mitte sich immer eine Königin befindet, die abgestochen wird.

\section{DISKUSSION}

Der Begriff Polygynie ist durch eine Koexistenz von Königinnen, gleichgültig ob sie besamt oder unbesamt sind, definiert. In der Ameisenliteratur gelten nur solche Kolonien als echt polygyn, die mehrere besamte eierlegende Königinnen aufweisen (Buschinger, 1974). Die von uns beobachtete Polygynie würde demzufolge als Pseudopolygynie eingestuft werden. Wir wollen aber der Bienenliteratur folgend 
weiterhin von Polygynie sprechen. Die sizilianische Honigbiene zeigt in ihrem Schwarmverhalten nur eine temporäre Polygynie: In den Schwärmen sind zwar keine Aggressionen zwischen den Bienen zu beobachten, aber im Volk und in den neuen Kolonien ändert sich dieses Verhalten mit einem Lebensalter der Königinnen von 2 Tagen. Die Königinnen werden aggressiv behandelt (Knäulen der Arbeiterinnen um die Königin) und abgestochen. Sicherlich sind sie dann auch untereinander aggressiver, denn die Freilandergebnisse decken sich genau mit Versuchen zur Überlebensdauer von Königinnen im Labor.

In Kästchenversuchen wurden Experimente zum Aggressionsverhalten der Königinnen untereinander durchgeführt (Methodik Tiemann et al, 1991). Königinnen im Alter von einem Tag und im Alter von 2 Tagen wurden mit 20 Begleitbienen auf ihre Überlebensdauer getestet. Es zeigten sich signifikante Unterschiede $(P \leq 0.05)$ in der Überlebensdauer. Königinnen im Alter von 2 Tagen haben ein deutlich höheres Aggressionsniveau als Königinnen im Alter von einem Tag (s Tiemann und Brückner, 1992). Vermutlich ist ein Anstieg eines oder mehrerer Pheromonspiegel der Grund für den Wechsel in der Aggressivität. Diese Ergebnisse stehen in Widerspruch zu den bisherigen Vorstellungen zum Reproduktionsverhalten $\operatorname{der} A m$ sicula. Es wurde eine Polygynie der neuen Kolonien bis nach den Paarungsflug der Königinnen vermutet (Ruttner, 1988; Engels, 1990). Die Monogynie war bei den von uns untersuchten Schwärmen in der Mehrzahl nach 2 bis 3 Tagen wiederhergestellt. Wenn in Ausnahmen Königinnen länger koexistierten, waren die Schwärme sehr groß, auch diese Kolonien waren nach 7 Tagen wieder monogyn. Im alten Volk ist auch keine langfristige Polygynie zu erwarten, da die Königinnen in $99 \%$ der Fälle nur eine Überlebensdauer von 2 Tagen zeigten.
Die sizilianische Honigbiene ist in ihrer Weiselzellentwicklung und Schwarmproduktion hoch aktiv. Dieses Reproduktionsverhalten erfordert eine hohe Produktivität der Futtersaftdrüsen der Arbeiterinnen und starke Vitalität der eierlegenden Königin. In 5 von 9 Fällen wurde die alte Königin von den Bienen vor dem Schwärmen abgestochen. Möglicherweise ist diese häufig gar nicht mehr in der Lage zu schwärmen, weil ihre Überlebensdauer durch die hohe Leistung in der ununterbrochenen Eiablage erschöpft ist oder weil sie nicht wie die nordeuropäische Rasse Carnica einen Brutstopp einlegt, damit sich das Abdomen verkürzt und sie abfliegen kann. Die erschöpfte Vitalität der noch schwärmenden alten Königinnen kann sich in einer geringeren Pheromonkonzentration äußern und würde die ungewöhnlich geringe Affinität der Bienen zur alten Königin im Vorschwarm erklären.

Zusammenfassend läßt sich sagen, daß das Schwarmverhalten der sizilianischen Honigbienenrasse im Gegensatz zu den anderen europäischen Rassen (Berlepsch, 1860; Zander und Weiß, 1964; Wiston, 1987) einige Besonderheiten zeigt:

- Diese Bienenrasse produziert in der Schwarmzeit eine enorme Anzahl an Weiselzellen.

- Es schlüpfen eine große Anzahl an Königinnen gleichzeitig, bis zu 33 täglich sind beobachtet worden.

- Die Königinnen koexistieren im Volk und in der neuen Kolonie in der Mehrzahl nur bis zu einem Lebensalter von 2 bis 3 Tagen.

- Alle Schwärme sind polygyn, fremde Königinnen werden ebenso toleriert wie fremde Arbeiterinnen.

- Das Volk produziert so viele Schwärme, daß es sich, bis auf ein kleines Restvolk, auflöst.

Interessant zu untersuchen wäre zum einen, welche Pheromonspiegel sich inner- 
halb der Rasse im Alter von 1 Tag zu 2 Tagen meßbar ändern, zum anderen, ob eine andere Pheromonkonzentration oder Zusammensetzung der Pheromone die Königinnen dieser Rasse zu den anderen europäischen Honigbienenrassen abgrenzt.

Das Reproduktionsverhalten der anderen polygynen Rassen ist ebenfalls wichtig zu überprüfen, da wahrscheinlich eine enge Verwandtschaft zu mindestens einer dieser Rassen besteht. Weiterführende Untersuchungen sind von uns geplant.

\section{DANKSAGUNG}

Für die Anregung zu diesem Therna und die Unterstützung im Verlauf der Untersuchungen danken wir Prof Dr F Ruttner. Zusätzlich danken wir Prof V Stampa für seine Gastfreundschaft und die Bereitstellung der Bienenvölker und $\mathrm{Dr}$ $\mathrm{H}$ Schmidt-Uhlenkamp für seine Mitarbeit und anregenden Diskussionen.

Die Arbeit wurde durch einen Reisekostenzuschuß der DFG gefördert. Diese Veröffentlichung ist Teil der Dissertation von Karin Tiemann.

\section{Summary - The swarming behavior of the Sicilian honeybee (Apis mellifera si-} cula). The reproductive behavior of this honeybee is different from all other European mellifera races. In the swarming season the colonies produce a high number of queen cells, and many virgin queens live together in the old and new colonies (Ruttner, 1988). In the spring of 1992, we investigated 9 bee hives near Trapani (western Sicily). The hives were supplied with extra food and were controlled daily as follows: the mother queens' intention to swarm was estimated by observing their egg production and motor activity. The new queens were marked individually by numbered opalith-platelets to determine their lifespan. In addition, we were able to investigate the reactions of the worker bees in the polygynous phase and the aggression between the queens. Two d after swarming, the queens were counted and their behavior in the new colony observed. As expected, the bee colonies showed a high production of queen cells, with a maximum of 232 capped cells in $1 \mathrm{~d}$ (see fig 1). The number of emerged queens varied from 1 to 33 individuals per d. In the old colonies the new queens were killed by workers or sister queens within $2 \mathrm{~d}$ (see fig 2). Many queens got lost during the swarming process. One prime swarm was observed; in 5 other colonies the mother queens were killed before swarming. This seems to be typical of the Sicilian honeybee. Worker bees showed no strong aggression to virgin queens in the old colony; even foreign queens that entered the hive were tolerated. In the swarm clusters the worker bees showed little interest towards individual queens. If queens fell out of the cluster, only a few bees reacted, and the queens were tolerated when they returned to the swarm. The entire swarm returned to the hive when $<3$ queens were in the cluster. Swarms can associate with other swarms, including foreign swarms, or can split into subswarms. In the new colonies we observed 2 to 13 coexisting queens (see fig 3 ). The duration of survival of the virgin queens was limited to 2 to $3 \mathrm{~d}$. Nearly $70 \%$ of the queens were killed during this period of time. Usually, the worker kees killed the queens by balling. Before the mating flights, which started at age 6-10 d for the queens, nearly all new colonies were again monogynous.

Survival of the new queens in old and new colonies was found to be in accordance with earlier laboratory tests. In these tests 2 virgin sicula queens were caged together with 20 worker bees (Tiemann et al, 1991). Queens at age $2 \mathrm{~d}$ showed a signifi- 
cantly higher aggression rate $(P \leq 0.05)$ than queens at age $1 \mathrm{~d}$ (Tiemann and Brückner, 1992). We assume that the change in aggression rate was caused by the increase in concentration of one or more pheromones.

It was thought that the Sicilian honeybee remained polygynous until the mating flights had been completed (Ruttner, 1988; Engels, 1990). We observed aggressive behavior between queens and workers independent of the mating flights and long before they took place. Further investigations are planned to determine which pheromones change within $2 \mathrm{~d}$ in the virgin queens and whether a special pheromone pattern exists in the Sicilian race which is different from that of other European races.

\section{Apis mellifera sicula / swarming behav- ior / polygyny}

\section{Résumé - Le comportement d'essai-} mage de l'abeille sicilienne, Apis mellifera sicula Montagano. Le comportement reproducteur de cette abeille diffère de celui de toutes les autres races européennes d'Apis mellifera. À la période de l'essaimage, les colonies produisent des cellules royales en grand nombre et de nombreuses reines vierges vivent ensemble dans les anciennes et les nouvelles colonies (Ruttner, 1988). Au printemps 1992, nous avons étudié 9 colonies situées près de Trapani (Sicile occidentale). La nourriture leur a été fournie ad libitum et les contrôles journaliers ont porté sur les paramètres suivants : l'intention des reines mères à essaimer a été estimée d'après leur production d'œufs et leur activité locomotrice. Les nouvelles reines ont été marquées individuellement avec des pastilles numérotées, afin de déterminer leur durée de vie. Nous avons pu en outre étudier les réactions des ouvrières pendant la phase de polygynie et l'agression entre les reines. Deux jours après l'essaimage, nous avons compté les reines et observé leur comportement dans la nouvelle colonie. Comme on s'y attendait, les colonies ont produit de nombreuses cellules royales, avec un maximum de 232 cellules operculées en 1 j (fig 1). Le nombre de reines émergeant par jour a varié de 1 à 33 . Dans les anciennes colonies, les nouvelles reines ont été tuées par les ouvrières ou les reines sœurs en l'espace de 2 j (fig 2). De nombreuses reines ont été perdues pendant l'essaimage. Un essaim primaire a été observé; dans 5 autres colonies les reines mères ont été tuées avant l'essaimage. Cela semble caractéristique de l'abeille sicilienne. Les ouvrières n'ont pas montré d'agression marquée vis-à-vis des reines vierges dans la vieille colonie; même les reines étrangères qui pénétraient dans la ruche étaient tolérées. Dans les essaims, les ouvrières montraient peu d'intérêt envers les reines. Si les reines tombaient de la grappe, seules quelques ouvrières réagissaient et les reines étaient tolérées lorsqu'elles retournaient dans l'essaim. L'essaim entier retournait à la ruche lorsqu'il comportait moins de 3 reines. Les essaims s'associaient à d'autres essaims, y compris étrangers, ou se scindaient en sous-essaims. Dans les nouvelles colonies, nous avons observé 2 à 13 reines qui coexistaient (fig 3 ). La durée de survie des reines vierges est limitée à 2 à 3 j. Près de $70 \%$ des reines ont été tuées durant cette période par les ouvrières qui les emballaient. Presque toutes les colonies sont redevenues monogynes avant les vols nuptiaux, qui débutent lorsque la reine est âgée de 6 à $10 \mathrm{j}$. La survie des nouvelles reines dans les anciennes et les nouvelles colonies concordent avec les tests faits précédemment au laboratoire. Dans ces tests, 2 reines vierges sicula étaient encagées ensemble avec 20 ouvrières (Tiemann et al, 1991). Les reines âgées de $2 \mathrm{j}$ 
présentaient un taux d'aggressivité nettement plus élevé $(P \leq 0,05)$ que les reines âgées d'1 j (Tiemann et Brückner, 1992). Nous pensons que ce changement est provoqué par l'accroissement de la concentration d'une ou plusieurs phéromones. On pensait jusqu'à présent que l'abeille sicilienne restait polygyne jusqu'au vol nuptial (Ruttner, 1988; Engels, 1990). Nous avons observé un comportement agressif entre des reines et des ouvrières, indépendamment des vols nuptiaux et longtemps avant qu'ils n'aient lieu. II est prévu d'autres recherches pour savoir quelles phéromones se modifient au cours des 2 premiers jours de la vie des reines et s'il existe, chez l'abeille sicilienne, un bouquet phéromonal spécial différent de celui des autres races européennes.

\section{Apis mellifera sicula / comportement d'essaimage / polygynie}

\section{LITERATURLISTE}

Berlepsch A (1860) Die Biene und die Bienenzucht, Verlag der Friedrich Heinrichshofenschen Buchhandlung, Mühlhausen Thüringen
Buschinger A (1974) Monogynie und Polygynie in Insektensozietäten. In: Sozialpolymorphismus bei Insekten (Schmidt GH, ed) Wissenschaftl. Verlagsgesell $\mathrm{mbH}$, Stuttgart, 862856

Buttel-Reepen H (1921) Zur Lebensweise der ägyptischen Biene (Apis mellifica fasciata Latr) Sowie einiges zur Geschichte der Bienenzucht, Arch Bienenkd 3, 19-67

Engels W (ed) (1990) Social Insects, An Evolutionary Approach to Castes and Reproduction. Springer Verlag, Berlin

Kauhausen-Keller D (1991) Biometrische Unterscheidung zwischen Apis mellifera carnica Poll und anderen Rassen von Apis mellifera L. Apidologie 22 (2), 97-103

Ruttner F (1988) Biogeography and Taxonomy of Honeybees. Springer-Verlag, Berlin

Tiemann K, Brückner D, Schmidt-Unlenkamp H (1991) Zur Biologie der sizilianischen Honigbiene (Apis mellifera sicula). Apidologie 22 (4), 477-479

Tiemann K, Brückner D (1992) Polygynie bei Apis m sicula - ein seltenes Merkmal bei der Honigbiene. 13. Tagung der Deutschsprachigen Sektion der Internationalen Union zum Studium der Sozialen Insekten (IUSSI)

Winston M (1987) The Biology of the Honey Bee. Harvard University Press, Cambridge, MA

Zander E, Weiß U (1964) Das Leben der Biene. Ulmer Verlag, Stuttgart 